\title{
Mitarbeiterinnen und Mitarbeiter
}

DOI 10.1515/mgzs-2016-0067

Afflerbach, Holger, Prof. Dr., University of Leeds, UK, H.H.W.Afflerbach@leeds.ac.uk 21

Bachinger, Bernhard, Ludwig Boltzmann Institut für Kriegsfolgenforschung Graz, bernhard.bachinger@bik.ac.at 134

Bähr, Karen, Gedenkstätte Bergen Belsen, karenbaehr@web.de 151

Beddies, Thomas, Prof. Dr., Charité - Universitätsmedizin Berlin, Institut für Geschichte der Medizin, Thielallee 71, 14195 Berlin, Tel: 030-450529241, Fax: 030-450529901, thomas.beddies@charite.de — 232

Bergien, Rüdiger, Dr., Zentrum für Zeithistorische Forschung, bergien@zzf-pdm.de 261

Bosch, Gabriele, Dr., Bibliotheksoberrätin, ZMSBw, Potsdam, GabrieleBosch@bundeswehr.org — 167; 291

Bruhn, Karen, M.A., Wissenschaftliche Mitarbeiterin, Christian-Albrechts-Universität zu Kiel, karenbruhn@gmx.net 123

Epkenhans, Michael, Prof. Dr., Leitender Wissenschaftler im ZMSBw, Potsdam, MichaelEpkenhans@bundeswehr.org — 241

Frommeld, Debora, M.A., Soziologin, Institut für Geschichte, Theorie und Ethik der Medizin, Universität Ulm, debora.frommeld@uni-ulm.de — 141

Ganseuer, Frank, Dr., Oldenburg, IFGanseuer@t-online.de — 193

Grosse, Cornelia, ZMSBw, Potsdam, CorneliaGrosse@bundeswehr.org 157

Haas, Philip, Dr. des., Philipps-Universität Marburg, Institut für Neuere und Neueste Geschichte - Frühe Neuzeit, haasph@staff.uni-marburg.de — 1 
Hasselhorn, Benjamin, Dr. mult., Kurator Nationale Sonderausstellung 2017, Stiftung Luthergedenkstätten in Sachsen-Anhalt, Lutherstadt Wittenberg, Benjamin.Hasselhorn@martinluther.de — 236

Hecker, Hans, Dr. phil., Univ.-Prof., Osteuropäische Geschichte, Hans.Hecker@t-online.de — 177; 180; 224

Heinemann, Winfried, Prof. Dr., Oberst, ZMSBw, Potsdam, WinfriedHeinemann@bundeswehr.org — 279; 280

Helmecke, Chris, ZMSBw, Potsdam, ChrisHelmecke@bundeswehr.org — 55

Herold, Heiko, Dr. phil., Historiker, Hamburg, Heiko_Herold@gmx.de — 238; 243; 317

Hieck, Leonie, KptLtn, ZMSBw, Potsdam, LeonieHieck@bundeswehr.org — 157 Himmelsbach, Andreas, M.A., Filderstadt, andreas.himmelsbach@googlemail.com — 287

Hofmann, Andreas R., Dr., Leipzig, arhofmann@klioman.de — 202; 219

Hofmeister, Björn, Dr., Freie Universität Berlin, bhofmeister@zedat.fu-berlin.de; b-hofmeister@web.de 257

Hosfeld, Rolf, Dr., wissenschaftlicher Leiter des Lepsiushauses in Potsdam, rolf.hosfeld@gmx.de 245

Kettmann, Marius, B.A., Leverkusen, marius.kettmann@web.de — 309

Koller, Christian, Prof. Dr. phil., Universität Zürich, Direktor des Schweizerischen Sozialarchivs, koller@sozarch.uzh.ch — 264; 265; 285; 294

Koop, Volker, Freier Journalist, Publizist, Berlin, volker.koop@t-online.de — 315

Kümper, Hiram, Prof. Dr., Historisches Institut, Universität Mannheim, Hiram.Kuemper@uni-mannheim.de — 189; 191

Lange, Sven, Dr., Oberst, USAWC, Carlisle, PA — 172 
Lein, Richard, Dr., Karl-Franzens-Universität Graz, richard.lein@uni-graz.at — 134

Lutsch, Andreas, M.A., Stanford University, Stanford, CA, andreas.lutsch@uni-wuerzburg.de — 312

Meier, Martin, Dr. phil., Warin, Martinmeier05@aol.com — 199; 208

Moll, Martin, Univ.-Doz. Dr., Graz, martin.moll@uni-graz.at — 182; 296

Overmans, Rüdiger, Dr. rer. pol. et phil., OTL a.D., Freiburg i.Br., mail@ruediger-overmans.de — 303

Plassmann, Max, Dr., Köln, Max.Plassmann@Stadt-Koeln.de —— 165; 212

Pöhlmann, Markus, Dr., Wiss. Dir., ZMSBw, Potsdam, MarkusPoehlmann@bundeswehr.org — 268

Popp, Peter Andreas, Dr. phil., OTL, Offizierschule der Luftwaffe, Fürstenfeldbruck, PeterPopp@bundeswehr.org — 229

Querengässer, Alexander, Leipzig — 170; 175

Reichherzer, Frank, Dr., ZMSBw, Potsdam,

FrankReichherzer@bundeswehr.org — 273

Rischke-Neß, Janine, M.A., Doktorandin Universität Potsdam, rischke@uni-potsdam.de — 205

von Salisch, Marcus, Dr. phil., marcus.von.salisch@gmx.de — 254

Scianna, Bastian Matteo, M.A., Universität Potsdam, scianna@uni-potsdam.de — 145

Searle, Alaric, Prof. Dr., Professor of Modern European History, School of Arts and Media, University of Salford, Salford M5 4WT, UK, d.a.searle@salford.ac.uk — 300

Söhner, Felicitas, Dr., Medizinhistorikerin, Institut für Geschichte, Theorie und Ethik der Medizin, Universität UIm, felicitas.soehner@uni-ulm.de 
Strohmeier, Denis, M.A., Berlin, denis.strohmeier@gmx.de — 282

Stübig, Heinz, Prof. i.R. Dr., Marburg, stuebig@staff.uni-marburg.de — 217; 222; 227

Terre, Emilie, ZMSBw, Potsdam, EmilieTerre@bundeswehr.org — 248

Tewes, Ludger, PD Dr., Potsdam, Itewes@uni-potsdam.de — 276

Theilig, Stephan, Dr., wissenschaftlicher Leiter Brandenburg-Preußen Museum Wustrau, Leiter des Instituts für Caucasica-, Tatarica- und Turkestan Studien, Berlin, stephan-theilig@web.de 197

Thoß, Bruno, Dr., Leitender Wiss. Dir. a.D., Birkenweg 7, 72488 Sigmaringen 305

Trifković, Gaj, Dr., Austrian Center for Intelligence, Propaganda and Security Studies (ACIPSS), Karl-Franzens-Universität, Graz, trifkovg@gmail.com — 94

Tritsch, Marie, Französische Botschaft, Berlin, marie.tritsch.ext@intradef.gouv.fr; tritsch@yahoo.fr — 185

Wunderlich, Sven, Lehramtsstudent und Studentische Hilfskraft, Universität Köln, sven.wunderlich@uni-koeln.de — 128

Wurzer, Georg, Dr. phil., Wilhelmsdorf (Württemberg), georgwurzer@gmx.de — 251 
